\title{
Measuring negative symptoms in patients with schizophrenia: reliability and validity of the Korean version of the Motivation and Pleasure Scale-Self-Report
}

\author{
This article was published in the following Dove Press journal: \\ Neuropsychiatric Disease and Treatment \\ II May 2016 \\ Number of times this article has been viewed
}

\author{
Ji-Sun Kim \\ Seon-Kyeong Jang' \\ Seon-Cheol Park ${ }^{2}$ \\ Jung-Seo $\mathrm{Yi}^{3}$ \\ Joong-Kyu Park ${ }^{4}$ \\ Jung Suk Lee ${ }^{5}$ \\ Kee-Hong Choi ${ }^{6}$ \\ Seung-Hwan Lee ${ }^{1,7}$ \\ 'Clinical Emotion and Cognition \\ Research Laboratory, Goyang, \\ ${ }^{2}$ Department of Psychiatry, Inje \\ University Haeundae Paik Hospital, \\ Busan, ${ }^{3}$ Department of Psychiatry, \\ Kangnam Sacred Heart Hospital, \\ Hallym University College of \\ Medicine, Seoul, ${ }^{4}$ Department of \\ Rehabilitation Psychology, Daegu \\ University, Daegu, ${ }^{5}$ Department of \\ Psychiatry, National Health Insurance \\ Service Ilsan Hospital, Goyang, \\ ${ }^{6}$ Department of Psychology, Korea \\ University, Seoul, ${ }^{7}$ Department of \\ Psychiatry, Ilsan Paik Hospital, Inje \\ University College of Medicine, \\ Goyang, Republic of Korea
}

Correspondence: Seung-Hwan Lee Department of Psychiatry, Ilsan Paik Hospital, Inje University College of Medicine, Juhwa-ro I70, Ilsanseo-Gu, Goyang 4I I-706, Republic of Korea $\mathrm{Tel}+82319107260$

Fax+82 31910 7268

Email ishpss@hanmail.net
Background: The Clinical Assessment Interview for Negative Symptoms (CAINS) is one of the validated interview measures of negative symptoms in psychotic disorders. The Motivation and Pleasure Scale-Self-Report (MPSR) is a self-report measure that assesses the motivation and pleasure domains of negative symptoms based on the CAINS. This study evaluated the reliability and validity of a Korean version of the MPSR.

Methods: A total of 139 patients with schizophrenia completed the MPSR, CAINS, Scale for the Assessment of Negative Symptoms (SANS), Brief Psychiatric Rating Scales, Calgary Depression Scale for Schizophrenia, and other measures of trait and cognitive function.

Results: The 15-item MPSR showed good internal consistency. In addition, it also had a good convergent validity with the Motivation and Pleasure subscale of the CAINS and the anhedonia/ avolition subscale of the SANS. The scale was not associated with psychotic symptoms, agitation/ mania, and depression/anxiety, and it showed good discriminant validity. MPSR scores were significantly correlated with Behavioral Activation System total score for trait measure.

Conclusion: The Korean version of the MPSR is a notable self-report method for examining the severity of negative symptoms in schizophrenia.

Keywords: Korean version of the MPSR, CAINS, negative symptoms, reliability, validity

\section{Introduction}

Negative symptoms of schizophrenia are clearly important issues for determining patients' quality of life, especially their social lives. ${ }^{1}$ The increasing number of researchers and clinicians are focusing their interests and putting their efforts on improving skills to provide appropriate management of negative symptoms for the patients. ${ }^{1}$ Negative symptoms are essential to the pathology of schizophrenia and are associated with significant decline of motivation, verbal and nonverbal communication, affect, and cognitive and social functioning. ${ }^{1,2}$ Therefore, the negative symptoms give a huge impact on poor outcome and functioning in schizophrenia. . $^{1,3,4}$ The importance of the negative symptoms accentuates the sound evaluation of the symptoms, ${ }^{5}$ and several assessment tools for negative symptoms have been developed. However, the most widely used negative symptom assessments, the Scale for the Assessment of Negative Symptoms (SANS) ${ }^{6}$ and the Positive and Negative Syndrome Scale, ${ }^{7}$ have some limitations. ${ }^{8}$ Some items from the two scales may be more related to cognitive functioning which has now been regarded to be conceptually distinct 
from negative symptoms, ${ }^{9-11}$ and a conceptual issue of the scales is the overlap with measures of functional outcome..$^{9,12}$ Additionally, the measurements mainly concentrate on observable symptoms and fail to evaluate internal experiences of patients. ${ }^{8,9,13}$

The Clinical Assessment Interview for Negative Symptoms (CAINS) was developed to complement the limitations with previous measures of negative symptoms. ${ }^{8,12-14}$ The CAINS has two factor-derived scales measuring loss of motivation and pleasure (MP) and expression (EXP). ${ }^{14,15}$

A self-report measure could save clinician's time with quicker initial assessment of the negative symptoms of patients. ${ }^{14,16}$ Additionally, a self-report measure could make patients more involved with their responses and treatment plans. It could enhance the therapeutic alliance and treatment outcome. ${ }^{17}$ Because negative symptoms are associated with patient's internal experiences, the self-report measure might be more suitable for assessment of patients' negative symptoms. With an increasing need for self-report tools for negative symptoms, the Motivation and Pleasure ScaleSelf-Report (MPSR) has been developed to assess the MP domain of negative symptoms based on the CAINS. ${ }^{14}$ The MPSR is a refined measurement focusing on the MP factor of CAINS by removing the EXP factor-related items of CAINS due to poor reliability and validity. ${ }^{14}$ The revised 15-item version of MPSR showed excellent internal consistency and good convergent and discriminant validity in a previous study. ${ }^{14}$ This study aims to evaluate the reliability and validity of a Korean version of the MPSR in patients with schizophrenia.

\section{Methods}

\section{Participants}

The total sample included 139 patients with schizophrenia recruited from outpatient clinics at four sites (Inje University Ilsan Paik Hospital, Yong-In Mental Hospital, Hallym University Kangnam Sacred Heart Hospital, Korea University). Diagnoses were made by psychiatrists using the Structured Clinical Interview for DSM-IV Axis I Disorders. ${ }^{18}$ Exclusion criteria included: 1) history of head injury or electroconvulsive treatment, 2) comorbid substance dependence or abuse, 3) serious medical conditions, and 4) low intelligence without the ability to understand or participate in the study. Duration of illness and number of hospitalizations were assessed. Demographic and clinical characteristics of participants are presented in Table 1. All participants provided written informed consent. The study was approved by the ethics committees of the Institutional Review Board at all four sites.
Table I Baseline demographic and clinical characteristics of participants $(\mathrm{N}=139)$

\begin{tabular}{ll}
\hline Characteristics & Mean (SD) or $\mathbf{n}(\%)$ \\
\hline Age (years), mean (SD) & $38.9(\mathrm{II} . \mathrm{I})$ \\
Sex, $\mathrm{n}(\%)$ & $76(54.7)$ \\
$\quad$ Male & $63(45.3)$ \\
$\quad$ Female & $13.1(\mathrm{I} .6)$ \\
Education (years), mean (SD) & $13.6(8.9)$ \\
Duration of illness (years), mean (SD) & $3.9(4.3)$ \\
Number of hospitalizations, mean (SD) & \\
BPRS, mean (SD) & $16.3(6.2)$ \\
$\quad$ Positive symptoms & $4.6(1.9)$ \\
$\quad$ Agitation/mania & $7.9(3.3)$ \\
$\quad$ Negative symptoms & $9.4(4.1)$ \\
Depression/anxiety & $4.9(4.9)$ \\
CDSS, mean (SD) & $14.0($ I3.5) \\
SANS (flat affect/alogia), mean (SD) & $18.3(7.6)$ \\
SANS (anhedonia/avolition), mean (SD) & $32.3(18.3)$ \\
SANS (total), mean (SD) &
\end{tabular}

Abbreviations: BPRS, Brief Psychiatric Rating Scale; CDSS, Calgary Depression Scale for Schizophrenia; SANS, Scale for the Assessment of Negative Symptoms; SD, standard deviation.

\section{Procedures}

The MPSR ${ }^{14}$ was translated into Korean by two researchers fluent in both English and Korean (SHL and JSL) with prior agreement from the developers of the original scale. A final consensus version was discussed with clinically experienced colleagues and was then back-translated into English by one translator (SHL). The translation was compared with the original English version, and no further changes were found to be necessary.

\section{Measures}

\section{Clinical symptom measures}

In addition to the MPSR, four more symptom measure scales were applied: CAINS, Brief Psychiatric Rating Scales (BPRS), SANS, and Calgary Depression Scale for Schizophrenia (CDSS). Negative symptoms were evaluated by the CAINS ${ }^{8,15}$ and SANS. ${ }^{6,19}$ The Korean version of CAINS (CAINS-K) is a 13-item semi-structured interview. The CAINS-K also has two factors like the original CAINS: EXP (four items) and MP (nine items), and has demonstrated good internal consistency ( $\alpha=0.89$ for EXP, 0.91 for MP). ${ }^{14}$ The CAINS-K demonstrated good convergent and discriminant validity in our previous study (Jang et al, unpublished data, 2016). The original SANS is composed of 19 items that are divided into five subscales including affective flattening, alogia, avolition-apathy, anhedonia-asociality, and attention. ${ }^{20}$ The SANS is regarded as a well-developed tool for negative symptom assessment, ${ }^{11}$ with good validity and reliability. ${ }^{6,19}$ The BPRS is a clinician-rated measure that 
assesses psychiatric symptoms such as somatic concern, suicidality, unusual thought content, suspiciousness, ${ }^{14,21,22}$ and the 18-item BPRS was used in this study. ${ }^{21}$ The Likert-type scale ranges from 1 (not present) to 7 (extremely severe). The BPRS has four subscale scores (Positive symptoms, Negative symptoms, Agitation/mania, Depression/anxiety) which were used to evaluate the current level of psychopathology. ${ }^{23}$ The Korean version of BPRS used in the present study has favorable reliability and validity. ${ }^{24,25}$ The CDSS is a nine-item interview to evaluate depressive symptoms for patients with schizophrenia. ${ }^{14,26}$ Four-point responses to each item range from 0 (absent) to 3 (severe) in the scale. The CDSS has good reliability and validity. ${ }^{27}$ The Korean version of CDSS used in this study has been standardized and has good validity. ${ }^{28}$

\section{Neurocognitive and trait measures}

For evaluation of neurocognition, Coding subtest, TrailMaking Test-B (TMT-B), and verbal fluency task were used to examine a potential relationship between the MPSR and neurocognition. The Coding subtest has been known to reflect processing speed, short-term visual memory, and visual-motor coordination in schizophrenia. ${ }^{29}$ Performance of TMT-B has been related with executive functions such as set-shifting ability. ${ }^{30}$ Lastly, verbal fluency task has been related with a disorganized semantic system, psychomotor speed, and fronto-striatal dysfunction. ${ }^{31-33}$

For trait measure, Behavioral Activation System (BAS) and Behavioral Inhibition System (BIS) were used in the study. The BIS and BAS questionnaires ${ }^{34}$ were used to measure self-reported dysregulation of BIS and BAS. BAS comprises three subscales (Fun seeking, Reward responsiveness, Drive) and BIS is related to inhibition, withdrawal, anxiety, and response to threat. ${ }^{34}$ The Korean version of BAS/BIS used in this study has been shown to have good reliability and competent validity. ${ }^{35}$

\section{Data analysis}

Cronbach's alpha coefficients were calculated to investigate the internal consistency of 18 MPSR items. Correlation analyses were implemented to assess the convergent validity of the MPSR with the MP scale of the CAINS and anhedonia/avolition subscale of SANS. Pearson's correlation coefficients were also calculated to explore the discriminant validity of the MPSR with the EXP scale of the CAINS, flat affect/alogia subscale of SANS, BPRS subscales, and CDSS. Correlation analyses were also conducted to examine the associations between the MPSR and scores on the neurocognitive and trait measures. All statistical analyses were performed using SPSS version 18.0 for Windows (SPSS Inc., Chicago, IL, USA).

\section{Results Internal consistency}

Cronbach's alpha of the 18-item Korean version of the MPSR was 0.90. All items except three (items 8, 10, and 12) showed acceptable item-total correlation coefficients from 0.357 to 0.773 . The item-total correlation coefficients of these three items were $0.051,0.139$, and 0.232 , respectively. These poorly performing three items were removed and the Cronbach's alpha of the revised 15 items of MPSR was 0.93 . Deletion of three items yielded higher Cronbach's alpha than when all items were retained. Therefore, these items were eliminated from the scale and all subsequent analyses were carried out using the remaining 15 items. The three items removed from the Korean version of the MPSR were identical to the original study of the MPSR. ${ }^{14}$

\section{Convergent and discriminant validity}

The results of the correlation analysis between 15 items of MPSR and other measures are presented in Table 2. The MPSR score was significantly correlated with MP subscale of the CAINS and anhedonia/avolition subscale scores of SANS. It was also significantly correlated with EXP subscale of CAINS.

MPSR scores were not significantly correlated with Positive symptoms, Depression/anxiety, and Agitation/mania subscale of BPRS. MPSR scores were not significantly correlated with depressive symptoms of CDSS.

Table 2 Convergent and discriminant validity: correlations between MPSR and clinician-rated CAINS, SANS, BPRS, and CDSS

\begin{tabular}{ll}
\hline Clinical symptom measures & MPSR (r) \\
\hline CAINS & \\
$\quad$ Motivation and Pleasure & $0.45^{* *}$ \\
Expression & $0.22^{*}$ \\
SANS & \\
Anhedonia/avolition & $0.45^{* *}$ \\
Flat affect/alogia & 0.06 \\
BPRS & \\
Positive & 0.06 \\
Agitation/mania & 0.002 \\
Depression/anxiety & 0.14 \\
CDSS & 0.09
\end{tabular}

Notes: *Correlation is significant at the 0.05 level; **orrelation is significant at the 0.00 I level.

Abbreviations: MPSR, Motivation and Pleasure Scale-Self-Report; CAINS, Clinical Assessment Interview for Negative Symptoms; SANS, Scale for the Assessment of Negative Symptoms; BPRS, Brief Psychiatric Rating Scale; CDSS, Calgary Depression Scale for Schizophrenia. 
Table 3 Correlations between MPSR and neurocognitive and trait measures

\begin{tabular}{ll}
\hline Cognitive and trait measures & MPSR (r) \\
\hline Coding & 0.15 \\
TMT-B (time, seconds) & -0.05 \\
Verbal fluency & 0.15 \\
BAS scale & $0.35^{* *}$ \\
Drive & $0.43^{* *}$ \\
Fun seeking & $0.27^{* *}$ \\
Reward responsiveness & $0.25^{* *}$ \\
BIS scale & 0.06 \\
\hline
\end{tabular}

Note: ${ }^{* *}$ Correlation is significant at the $0.00 \mathrm{I}$ level.

Abbreviations: MPSR, Motivation and Pleasure Scale-Self-Report; TMT-B, Trail Making Test part B; BAS, Behavioral Activation System; BIS, Behavioral Inhibition System.

\section{Effects of age and sex}

Correlations between MPSR scores and age were assessed. MPSR scores were not significantly correlated with ages of participants $(P=0.16)$. Independent samples $t$-tests were conducted to test whether there was sex difference on MPSR scores, and the results showed that there was no sex difference on MPSR scores $(P=0.93)$.

\section{Correlations with neurocognitive and trait measures}

Correlations between MPSR scores and Coding subtest, TMT-B, BAS, and BIS are presented in Table 3. MPSR scores were not significantly correlated with neurocognitive measures such as Coding subtest, TMT-B, and verbal fluency test. With respect to trait measures, the MPSR was significantly correlated with BAS scores and its three subscales (Drive, Fun seeking, Reward responsiveness), but not with BIS scales.

\section{Discussion}

This study evaluated the reliability and validity of the Korean version of the MPSR in a large sample of individuals with schizophrenia. The Korean version of the MPSR is a selfreporting assessment tool for decline of MP that is one of the distinguished characteristics of negative symptoms in patients with schizophrenia.

In the present study, the Korean version of MPSR showed excellent internal consistency as in the original version of the MPSR. ${ }^{14}$ We removed three items which presented low itemtotal correlations as in a previous study by Llerena et al. ${ }^{14}$ These items of poor performance might be attributed to the reversed direction of presented sentences and it might have been a distraction to the participants. ${ }^{14}$ The remaining 15-item version also showed outstanding internal consistency in this study.

The MPSR showed good convergent validity with the MP subscale on the CAINS and anhedonia/avolition subscale of SANS. Not corresponding to the previous study, ${ }^{14}$ the MPSR showed significant partial correlations with the CAINS EXP scale. This unexpected result might be due to different characteristics among the subjects used in the studies. The age of Llerena et al's study population was older than that of our study, and therefore the illness duration might be longer than that of ours, although their study did not report the illness duration of the participants. Moreover, our study included more females than males compared to Llerena et al's study. ${ }^{14}$ Further studies are needed to examine whether this unexpected correlation is replicable.

The discriminant validity of the MPSR was also satisfactory. The MPSR was not significantly correlated with depressive symptoms or with whole subscales of the BPRS (the Positive symptoms or Depression/anxiety or Agitation/ mania subscales). These results were comparable to the studies investigating the clinician-administered CAINS MP subscale $^{36}$ and a preliminary study of a self-report version of the CAINS. ${ }^{37}$ As expected, the Korean version of the MPSR was not associated with the Agitation/mania subscale of the BPRS. In contrast, Llerena et al's study ${ }^{14}$ reported that the MPSR was correlated with the Agitation/mania subscale of the BPRS. One possible explanation for this discrepancy could be the characteristics of the participants in the two studies. Future studies would be needed to verify the association between the MPSR and the subscales of the BPRS.

MPSR scores were not related to age, sex, or neurocognitive function in the present study. In a previous study, Park et al reported that general cognitive ability was not correlated with CAINS Experiential (avolition, anhedonia, asociality) subscale. ${ }^{37}$ Also, there was no association between the MPSR and cognitive ability. ${ }^{14}$ Although our neurocognitive measure was not same as the previous two studies, the neurocognition measures examined in our study were related to processing speed, short-term visual memory, executive function, and visual-motor coordination, which have been demonstrated to be prominent deficient domains in schizophrenia. ${ }^{38,39}$ In line with the previous results, ${ }^{14,37}$ the Korean version of the MPSR showed good discriminant validity.

Moreover, the present study and a previous study ${ }^{36}$ found that the MPSR was correlated with BAS, not BIS. BAS has been considered to control approach behavior reacting to cues of reward via dopaminergic activity in the mesolimbic system..$^{40,41}$ Reduced dopaminergic sensitivity in 
the striatum is connected to negative symptoms ${ }^{42}$ and may diminish motivational and emotional responses to reward related situations. ${ }^{43,44}$ Therefore, lower sensitivity to reward (lower BAS) with high levels of negative symptoms could be expected. ${ }^{45}$ Because the MPSR focused on the negative symptoms associated with MP, it could be correlated with the BAS, not BIS. Given that the behavioral trait can be assessed well by self-reported measurements like BIS/BAS, the MPSR could be a proper tool to assess the relationship between the trait and negative symptoms.

This study has some limitations. First, other forms of self-reports regarding motivation or pleasure such as the Revised Social Anhedonia Scale or the Social Closeness Scale of the Multidimensional Personality Questionnaire used in Llerena et al's study ${ }^{14}$ were not available. To verify the MPSR's unique associations with negative symptoms as a self-report measurement, examining the association with other forms of self-reports would be needed in future studies. Second, no functioning measures were evaluated in this study. One previous study reported that the original version of the MPSR was related to social network relationship in schizophrenia. ${ }^{14}$ Regarding the importance of functioning in patients with schizophrenia, future studies should examine whether this relationship is replicable. This study had a relatively larger sample size than the study for the original version of the MPSR, but the symptom severity was similar to that of the previous one. Future research is needed to mention the issues about possibilities of the MPSR as a tool for evaluating patients with high levels of negative symptoms.

\section{Conclusion}

This study revealed that the Korean version of the MPSR is a reliable and valid self-report measure of negative symptoms in schizophrenia. The Korean version of the MPSR was validated on the largest sample since the original study, and extended the validation of the measure to the Korean population. This self-report form for negative symptoms could enhance treatment outcome by improving clinician's understanding of internal experiences of patients and facilitating patients' involvement in their treatment plans.

\section{Acknowledgments}

This work was supported by a grant from the Korea Science and Engineering Foundation (KOSEF), funded by the Korean government (NRF-2015R1A2A2A01003564), and is supported by the Lee Byoung Yoon Fund (2014).

\section{Disclosure}

The authors report no conflicts of interest in this work.

\section{References}

1. Bobes J, Arango C, Garcia-Garcia M, Rejas J. Prevalence of negative symptoms in outpatients with schizophrenia spectrum disorders treated with antipsychotics in routine clinical practice: findings from the CLAMORS study. J Clin Psychiatry. 2010;71(3):280-286.

2. Lindenmayer JP, Khan A, Iskander A, Abad MT, Parker B. A randomized controlled trial of olanzapine versus haloperidol in the treatment of primary negative symptoms and neurocognitive deficits in schizophrenia. J Clin Psychiatry. 2007;68(3):368-379.

3. Buckley PF, Stahl SM. Pharmacological treatment of negative symptoms of schizophrenia: therapeutic opportunity or cul-de-sac? Acta Psychiatr Scand. 2007;115(2):93-100.

4. Rabinowitz J, Levine SZ, Garibaldi G, Bugarski-Kirola D, Berardo CG, Kapur S. Negative symptoms have greater impact on functioning than positive symptoms in schizophrenia: analysis of CATIE data. Schizophr Res. 2012;137(1-3):147-150.

5. Dollfus S, Mach C, Morello R. Self-evaluation of negative symptoms: a novel tool to assess negative symptoms. Schizophr Bull. Epub 2015 Nov 12.

6. Andreasen NC. Negative symptoms in schizophrenia. Definition and reliability. Arch Gen Psychiatry. 1982;39(7):784-788.

7. Kay SR, Fiszbein A, Opler LA. The positive and negative syndrome scale (PANSS) for schizophrenia. Schizophr Bull. 1987;13(2): 261-276.

8. Blanchard JJ, Kring AM, Horan WP, Gur R. Toward the next generation of negative symptom assessments: the collaboration to advance negative symptom assessment in schizophrenia. Schizophr Bull. 2011; 37(2):291-299.

9. Engel M, Fritzsche A, Lincoln TM. Validation of the German version of the Clinical Assessment Interview for Negative Symptoms (CAINS). Psychiatry Res. 2014;220(1-2):659-663.

10. Harvey PD, Koren D, Reichenberg A, Bowie CR. Negative symptoms and cognitive deficits: what is the nature of their relationship? Schizophr Bull. 2006;32(2):250-258.

11. Kirkpatrick B, Fenton WS, Carpenter WT Jr, Marder SR. The NIMHMATRICS consensus statement on negative symptoms. Schizophr Bull. 2006;32(2):214-219.

12. Forbes C, Blanchard JJ, Bennett M, Horan WP, Kring A, Gur R. Initial development and preliminary validation of a new negative symptom measure: the Clinical Assessment Interview for Negative Symptoms (CAINS). Schizophr Res. 2010;124(1-3):36-42.

13. Horan WP, Kring AM, Blanchard JJ. Anhedonia in schizophrenia: a review of assessment strategies. Schizophr Bull. 2006;32(2):259-273.

14. Llerena K, Park SG, McCarthy JM, Couture SM, BennettME, Blanchard JJ. The Motivation and Pleasure Scale-Self-Report (MAP-SR): reliability and validity of a self-report measure of negative symptoms. Compr Psychiatry. 2013;54(5):568-574.

15. Horan WP, Kring AM, Gur RE, Reise SP, Blanchard JJ. Development and psychometric validation of the Clinical Assessment Interview for Negative Symptoms (CAINS). Schizophr Res. 2011;132(2-3): $140-145$.

16. Iancu I, Poreh A, Lehman B, Shamir E, Kotler M. The Positive and Negative Symptoms Questionnaire: a self-report scale in schizophrenia. Compr Psychiatry. 2005;46(1):61-66.

17. Eisen SV, Dickey B, Sederer LI. A self-report symptom and problem rating scale to increase inpatients' involvement in treatment. Psychiatr Serv. 2000;51(3):349-353.

18. First M, Spitzer, RL, Gibbon M, Williams JB. Structured Clinical Interview for the DSM-IV Axis I Disorders. New York, NY: New York State Psychiatric Institute, Biometrics Research Department; 1995.

19. Andreasen NC, Flaum MA. Schizophrenia and related psychotic disorders. Hosp Community Psychiatry. 1990;41(9):954-956. 
20. Lyne J, Renwick L, Grant T, et al. Scale for the assessment of negative symptoms structure in first episode psychosis. Psychiatry Res. 2013; 210(3):1191-1197.

21. Overall JE, Gorham DR. The Brief Psychiatric Rating Scale. Psychol Rep. 1962;10:779-812.

22. Ventura J, Nuechterlein KH, Subotnik KL, Gutkind D, Gilbert EA. Symptom dimensions in recent-onset schizophrenia and mania: a principal components analysis of the 24-item Brief Psychiatric Rating Scale. Psychiatry Res. 2000;97(2-3):129-135.

23. Kopelowicz A, Ventura J, Liberman RP, Mintz J. Consistency of Brief Psychiatric Rating Scale factor structure across a broad spectrum of schizophrenia patients. Psychopathology. 2008;41(2):77-84.

24. Andersen J, Larsen JK, Schultz V, et al. The Brief Psychiatric Rating Scale. Dimension of schizophrenia - reliability and construct validity. Psychopathology. 1989;22(2-3):168-176.

25. Park SC, Ostergaard SD, Choi J, et al. Is the BPRS-5 subscale of the psychotic depression assessment scale a reliable screening tool for psychotic depression? Results from the CRESCEND study. J Affect Disord. 2015;174:188-191.

26. Addington D, Addington J, Schissel B. A depression rating scale for schizophrenics. Schizophr Res. 1990;3(4):247-251.

27. Addington D, Addington J, Maticka-Tyndale E, Joyce J. Reliability and validity of a depression rating scale for schizophrenics. Schizophr Res. 1992;6(3):201-208.

28. Kim SW, Kim SJ, Yoon BH, et al. Diagnostic validity of assessment scales for depression in patients with schizophrenia. Psychiatry Res. 2006; 144(1):57-63.

29. Wechsler D. Wechsler Adult Intelligence Scale-Fourth Edition (WAIS-IV): Administration and scoring manual. San Antonia, TX: Pearson; 2008.

30. Reitan RM, Wolfson D. The Halstead-Reitan Neuropsychological Test Battery: Therapy and Clinical Interpretation. Tucson, AZ: Neuropsychological Press; 1985.

31. Bowie CR, Harvey PD, Moriarty PJ, Parrella M, White L, Davis KL. A comprehensive analysis of verbal fluency deficit in geriatric schizophrenia. Arch Clin Neuropsychol. 2004;19(2):289-303.

32. Joyce EM, Collinson SL, Crichton P. Verbal fluency in schizophrenia: relationship with executive function, semantic memory and clinical alogia. Psychol Med. 1996;26(1):39-49.
33. van Beilen M, Pijnenborg M, van Zomeren EH, van den Bosch RJ, Withaar FK, Bouma A. What is measured by verbal fluency tests in schizophrenia? Schizophr Res. 2004;69(2-3):267-276.

34. Carver CS, White TL. Behavioral inhibition, behavioral activation, and affective responses to impending reward and punishment: the BIS/BAS scales. J Pers Soc Psychol. 1994;67:319-333.

35. Kim KH, Kim WS. Korean-BAS/BIS Scale. Korean J Health Psychol. 2001;6 (2):19-37.

36. Kring AM, Gur RE, Blanchard JJ, Horan WP, Reise SP. The Clinical Assessment Interview for Negative Symptoms (CAINS): final development and validation. Am J Psychiatry. 2013;170(2):165-172.

37. Park SG, Llerena K, McCarthy JM, Couture SM, Bennett ME, Blanchard JJ. Screening for negative symptoms: preliminary results from the selfreport version of the Clinical Assessment Interview for Negative Symptoms. Schizophr Res. 2012;135(1-3):139-143.

38. Keefe RS. Cognition and motivation as treatment targets in schizophrenia. JAMA Psychiatry. 2014;71(9):987-988.

39. Schaub A, Neubauer N, Mueser KT, Engel R, Moller HJ. Neuropsychological functioning in inpatients with major depression or schizophrenia. BMC Psychiatry. 2013;13:203.

40. Depue RA, Collins PF. Neurobiology of the structure of personality: dopamine, facilitation of incentive motivation, and extraversion. Behav Brain Sci. 1999;22(3):491-517; discussion 518-569.

41. Gray JA. Brain systems that mediate both emotion and cognition. Cognition and Emotion. 1990;4(3):269-288.

42. Heinz A, Knable MB, Coppola R, et al. Psychomotor slowing, negative symptoms and dopamine receptor availability - an IBZM SPECT study in neuroleptic-treated and drug-free schizophrenic patients. Schizophr Res. 1998;31(1):19-26.

43. Robbins TW, Everitt BJ. Neurobehavioural mechanisms of reward and motivation. Curr Opin Neurobiol. 1996;6(2):228-236.

44. Schultz W, Dayan P, Montague PR. A neural substrate of prediction and reward. Science. 1997;275:1593-1599.

45. Scholten MR, van Honk J, Aleman A, Kahn RS. Behavioral inhibition system (BIS), behavioral activation system (BAS) and schizophrenia: relationship with psychopathology and physiology. J Psychiatr Res. 2006;40(7):638-645.
Neuropsychiatric Disease and Treatment

\section{Publish your work in this journal}

Neuropsychiatric Disease and Treatment is an international, peerreviewed journal of clinical therapeutics and pharmacology focusing on concise rapid reporting of clinical or pre-clinical studies on a range of neuropsychiatric and neurological disorders. This journal is indexed on PubMed Central, the 'PsycINFO' database and CAS,

\section{Dovepress}

and is the official journal of The International Neuropsychiatric Association (INA). The manuscript management system is completely online and includes a very quick and fair peer-review system, which is all easy to use. Visit http://www.dovepress.com/testimonials.php to read real quotes from published authors. 